\title{
Characterization of a group-norm by maximum functional equation and stability results
}

\author{
Muhammad Sarfraz ${ }^{\mathrm{a}}$, Fawad Ali ${ }^{\mathrm{b}}$, Qi Liu ${ }^{\mathrm{a}}$, Yongjin Li ${ }^{\mathrm{a}, *}$ \\ a School of Mathematics, Sun Yat-sen University, Guangzhou 510275 China \\ b School of Mathematics and Statistics, Xi'an Jiaotong University, Xi'an 710049 China
}

*Corresponding author, e-mail: stslyj@mail.sysu.edu.cn

Received 3 May 2021

Accepted 24 Jun 2021

ABSTRACT: Let $(G,\|\cdot\|,+)$ be a normed group, where $\|\cdot\|: G \rightarrow \mathbb{R}$. We study the equation

$$
\max \{\|x+y\|,\|x-y\|\}=\|x\|+\|y\| \quad \text { for all } x, y \in G .
$$

Without a commutativity assumption of the normed group $G$, we analyze the stability results and characterization of a group-norm by the given equation.

KEYWORDS: normed group, discretely normed abelian group, Tabor weakly commutative, stability

MSC2010: 20D60 54E35 11T71

\section{INTRODUCTION}

Simon et al [4] gave the characterization $f(g)=$ $|\eta(g)|$ for an additive function $\eta: G \rightarrow \mathbb{R}$ such that $\eta\left(g_{1}+g_{2}\right)=\eta\left(g_{1}\right)+\eta\left(g_{2}\right)$, which fulfills the equations

$$
\begin{aligned}
& \max \left\{f\left(g_{1}-g_{2}\right), f\left(g_{1}+g_{2}\right)\right\}=f\left(g_{1}\right)+f\left(g_{2}\right), \\
& \min \left\{f\left(g_{1}-g_{2}\right), f\left(g_{1}+g_{2}\right)\right\}=\left|f\left(g_{1}\right)-f\left(g_{2}\right)\right|
\end{aligned}
$$

for all $g_{1}, g_{2} \in G$, assuming that the domain of $f$ is an abelian group $G$. However, according to the stability results of Przebieracz [9], (2) is stable, and she presented a general theorem that proves the stability of (2), where $f: \mathbb{R} \rightarrow \mathbb{R}$ is considered as a continuous function of real variables.

Gilanyi et al [5] took into account the generalized version of (1), that is

$$
\max \left\{f\left(\left(g_{1} g_{2}\right) g_{2}\right), f\left(g_{1}\right)\right\}=f\left(g_{1} g_{2}\right)+f\left(g_{2}\right)
$$

for all $g_{1}, g_{2} \in G$, and demonstrated its stability for the real-valued function $f: G \rightarrow \mathbb{R}$ under the assumption of left identity, where $G$ is considered as a square-symmetric groupoid. Consequently, Volkmann [15] gave the generalization of (1) under the condition that $f\left(g_{1} g_{2} g_{3}\right)=f\left(g_{1} g_{3} g_{2}\right)$ holds for all $g_{1}, g_{2}, g_{3} \in G$. Stability results in connection with generalization of (1) can be found in [12], while a generalized version of (1) without commutativity condition can be seen in [14].
Furthermore, Redheffer in a joint paper with Volkmann [10] gave the solution to a Pexiderized version of (1),

$$
f(x)+g(y)=\max \{h(x-y), h(x+y)\}
$$

for all $x, y \in G$, where $f, g$ and $h$ are mappings from an abelian group $(G,+)$ to $\mathbb{R}$.

Since group-norms play an important role in establishing relation between norms and group structures; therefore, in the next section, it will be shown that the proposed equation

$$
\|x\|+\|y\|=\max \{\|x+y\|,\|x-y\|\}
$$

for all $x, y \in G$, characterizes the group-norm. Therefore, we established a reliable relation between normed-groups and functional equation (5) through the characterization of a group-norm function from a normed group $(G,\|\cdot\|,+)$ to $\mathbb{R}_{\geqslant 0}$ defined by $\|x\|:=|x|$ for all $x \in G$. A presentation of our proposed definition in the form of group-norm equivalent to (5) was investigated in [5] as

$$
\max \left\{\left\|\left(g_{1} g_{2}\right) g_{2}\right\|,\left\|g_{1}\right\|\right\}=\left\|g_{1} g_{2}\right\|+\left\|g_{2}\right\|
$$

for all $g_{1}, g_{2} \in G$.

The last section is devoted to the stability results of (5), where $(G,\|\cdot\|,+)$ is a normed group. Moreover, we will analyze the stability of (5) for a realvalued function defined on a normed group $G$. As a 
consequence of our main stability theorem of (5), we obtain the stability results of (5) on a Tabor weakly commutative group.

\section{ANALYSIS OF (5)}

Throughout this article, our normed group $G$ will in general be $(G,\|\cdot\|,+)$, and 0 is considered to be the neutral element unless otherwise stated.

Definition 1 Let $(G,+)$ be a group with the neutral element 0 , then we say its norm $\|\cdot\|: G \rightarrow[0, \infty)$ is called a group-norm if, for any $a, b \in G$, it fulfills the following properties:

(i) $\|a+b\| \leqslant\|a\|+\|b\|$;

(ii) $\|a\| \geqslant 0$, with $\|a\|=0$ if $a=0$;

(iii) $\|-a\|=\|a\|$.

If (i) and (ii) are satisfied, then the norm $\|\cdot\|$ is known as a pre-norm; if only (i) holds, then we say the norm $\|\cdot\|$ is a semi-norm. For instance, see $[2,13]$. A normed group is denoted by $(G,\|\cdot\|,+)$ where $\|\cdot\|$ is a group-norm and $(G,+)$ is a group.

Theorem 1 Suppose that $(G,+)$ is a group. A mapping $\|\cdot\|: G \rightarrow \mathbb{R}$ satisfies (5) if and only if $(G,\|\cdot\|,+)$ is a normed group.

Proof: Suppose that (5) holds. Setting $x=0$, we can compute $\|0\|+\|0\|=\max \{\|0\|,\|0\|\}=\|0\|$, which implies that $\|0\|=0$.

Also given condition yields that $\|x\|+\|x\|=$ $\max \{\|x+x\|,\|x-x\|\}=\max \{\|2 x\|,\|0\|\} \geqslant 0$, which gives that $2\|x\| \geqslant 0$, so $\|x\| \geqslant 0$. Since $\|x\| \geqslant 0$ and $\|x\|=0$ whenever $x=0$, then we can see $\|2 x\| \geqslant 0=\|0\|$, so we have $\|2 x\| \geqslant\|x-x\|$, then

$$
2\|x\|=\|x\|+\|x\|=\max \{\|x-x\|,\|2 x\|\}=\|2 x\|,
$$

therefore $2\|x\|=\|2 x\|$.

Moreover, setting $y=-x$ in (5) implies that $\|x\|+\|-x\|=\max \{\|x+x\|,\|x-x\|\}=$ $\max \{\|0\|,\|2 x\|\}=\|2 x\|=2\|x\|$, which gives that $\|-x\|=\|x\|$. Furthermore, we can observe from (5) that $\|x-y\| \leqslant\|x\|+\|y\|$ or $\|x+y\| \leqslant\|x\|+\|y\|$; hence, in either case, the triangle inequality holds. Hence $(G,\|\cdot\|,+)$ is a normed group. Conversely, let $G$ be a normed group defined by the group-norm $\|\cdot\|$. Obviously, $\|x\|+\|y\|=\max \{\|x-y\|,\|x+y\|\}$ for any $x, y \in G$.

Corollary 1 For a normed group $(G,\|\cdot\|,+)$, a groupnorm $\|\cdot\|: G \rightarrow \mathbb{R}$ fulfilling (5) is a conjugation and abelian group-norm.
Proof: Let $x, y \in G$, then the proof of conjugation group-norm consists of the following simple computation:

$$
\begin{aligned}
\|y\|+\|- & y+x+y \| \\
& =\max \{\|y-y+x+y\|,\|y-y-x+y\|\} \\
& =\max \{\|x+y\|,\|-x+y\|\} \\
& =\max \{\|-y-x\|,\|-y+x\| \|\} \\
& =\max \{\|-y+x\|,\|-y-x\|\} \\
& =\|-y\|+\|x\| \\
& =\|y\|+\|x\|,
\end{aligned}
$$

Therefore, $\|-y+x+y\|=\|x\|$. Writing $y+x$ instead of $x$, we can obtain that

$$
\|y+x\|=\|-y+y+x+y\|=\|x+y\|,
$$

which implies that $\|x+y\|=\|y+x\|$ for any $x, y \in$ $G$. Thus, the group-norm is abelian.

Theorem 2 Let $(G,\|\cdot\|,+)$ be a normed group then $\min \{\|x-y\|,\|x+y\|\} \leqslant|\|x\|-\|y\||$ holds for all $x, y \in G$.

Proof: Since $(G,\|\cdot\|,+)$ is a normed group, a groupnorm $\|\cdot\|: G \rightarrow \mathbb{R}$ satisfies (5). Making use of the conjugation group-norm, we first compute that

$$
\begin{aligned}
2\|x\| & =\|x\|+\|x\| \\
& =\|x\|+\|-y+x+y\| \\
& =\max \{\|x-y+x+y\|,\|x-y-x+y\|\} \\
& \geqslant\|x-y+x+y\| .
\end{aligned}
$$

Then we can obtain the required result by the following simple calculation:

$$
\begin{aligned}
& \max \{\|x-y\|,\|x+y\|\}+\min \{\|x-y\|,\|x+y\|\} \\
& \quad=\|x-y\|+\|x+y\| \\
& \|x\|+\|y\|+\min \{\|x-y\|,\|x+y\|\}=\|x-y\|+\|x+y\| \\
& \min \{\|x-y\|,\|x+y\|\}=\|x-y\|+\|x+y\|-\|x\|-\|y\| \\
& \quad=\max \{\|x-y+x+y\|,\|x-y-y-x\|\}-\|x\|-\|y\| \\
& \quad=\max \{\|x-y+x+y\|,\|x-2 y-x\|\}-\|x\|-\|y\| \\
& \quad \leqslant \max \{2\|x\|,\|-2 y\|\}-\|x\|-\|y\| \\
& \quad=\max \{2\|x\|, 2\|y\|\}-\|x\|-\|y\| \\
& \quad=2 \max \{\|x\|,\|y\|\}-\|x\|-\|y\| \leqslant|\|x\|-\|y\|| .
\end{aligned}
$$

By adding a certain condition for Theorem 2, we can extend the proof to remove the inequality. The following definition will play a key role in the proof of Theorem 3. 
Definition 2 ([7]) For a normed group $(G,\|\cdot\|,+)$, we say a mapping $\|\cdot\|: G \rightarrow \mathbb{R}$ satisfies condition (C) if

$$
\|u+z+v\|=\|u+v+z\| \quad \text { for any } u, z, v \in G .
$$

Obviously, any normed group $(G,\|\cdot\|,+)$ fulfills the proposed condition (C) whenever the group $G$ is abelian.

Theorem 3 Suppose that $(G,+)$ is a group and a mapping $\|\cdot\|: G \rightarrow \mathbb{R}$ fulfills the given condition (C), then $(G,\|\cdot\|,+)$ is a normed group if and only if the group-norm is 2-homogeneous, and also

$$
\min \{\|x-y\|,\|x+y\|\}=|\|x\|-\|y\||
$$

holds for all $x, y \in G$.

Proof: If $(G,\|\cdot\|,+)$ is a normed group, then it is obvious that $\|0\|=0$ holds and also $\|x\| \geqslant 0$ for any $x \in G$. Also we can obtain that $\|x\|+\|y\|=$ $\max \{\|x-y\|,\|x+y\|\}$; therefore, setting $x=y=0$, we can compute that $\|2 x\|=2\|x\|$, i.e., group-norm is 2-homogeneous. The following simple computation gives the proof of (7):

$$
\begin{aligned}
& \min \{\|x-y\|,\|x+y\|\}+\|x\|+\|y\| \\
& \quad=\min \{\|x-y\|,\|x+y\|\}+\max \{\|x-y\|,\|x+y\|\} \\
& \quad=\|x-y\|+\|x+y\| \\
& \quad=\max \{\|x+y+x-y\|,\|x+y+y-x\|\} \\
& \quad=\max \{\|x+y+x-y\|,\|x+2 y-x\|\} \\
& \quad=\max \{\|2 x\|,\|2 y\|\} \\
& \min \{\|x-y\|,\|x+y\|\}=\max \{2\|x\|, 2\|y\|\}-\|x\|-\|y\| \\
& \min \{\|x-y\|,\|x+y\|\}=|\|x\|-\|y\|| .
\end{aligned}
$$

Conversely, assume that a group-norm fulfills (7) and also is 2-homogeneous. Then we can see that

$$
\begin{array}{r}
\max \{\|x-y\|,\|x+y\|\}-\min \{\|x-y\|,\|x+y\|\} \\
=|\|x+y\|-\|x-y\|| .
\end{array}
$$

Applying (7) in the following computation, we obtain that

$$
\begin{aligned}
& \max \{\|x-y\|,\|x+y\|\}-|\|x\|-\|y\||=|\|x+y\|-\|x-y\|| \\
& \quad=\min \{\|x+y+x-y\|,\|x+y+y-x\|\} \\
& \quad=\min \{\|2 x\|,\|x+2 y-x\|\} \\
& \quad=\min \{\|2 x\|,\|2 y\|\} \\
& \max \{\|x-y\|,\|x+y\|\}=2 \min \{\|x\|,\|y\|\}+\mid\|x\|-\|y\| \| \\
& \max \{\|x-y\|,\|x+y\|\}=\|x\|+\|y\|,
\end{aligned}
$$

which implies that $(G,\|\cdot\|,+)$ is a normed group.
Corollary 2 If $(G,+)$ is a group and a mapping $\|\cdot\|: G \rightarrow \mathbb{R}$ fulfills the proposed condition (C), then $(G,\|\cdot\|,+)$ is a normed group if and only if

$$
\|x\|+\|y\|=\|x-y\|+\|x+y\|-|\|x\|-\|y\||,
$$

for any $x, y \in G$, and also $\|0\|=0$ holds.

Proof: Assume that (8) holds and also $\|0\|=0$. Then (8) yields that

$$
2 \max \{\|x\|,\|y\|\}=\|x-y\|+\|x+y\|, \quad x, y \in G .
$$

Since $\|0\|=0$, replacing $y$ with $x$, we can easily compute that $\|2 x\|=2\|x\|$. Then replacing $x$ with $x+y$ and $y$ with $x-y$ in (9), we have

$$
\begin{aligned}
2 \max \{\|x-y\|,\|x+y\|\} & =\{\|2 x\|+\|2 y\|\} \\
& =\{2\|x\|+2\|y\|\} \\
\max \{\|x-y\|,\|x+y\|\} & =\|x\|+\|y\| .
\end{aligned}
$$

Conversely, suppose $(G,\|\cdot\|,+)$ is a normed group, then, by Theorem 3 , we can determine that $\|0\|=0$, and (8) also holds.

\section{STABILITY OF (5)}

To analyze the stability results of (5) involving variables $x$ and $y$, first replacing $y$ with $x$ in (5), we will find the stability result of (5) in a single variable $x$ in the following theorem.

Theorem 4 Suppose that $(G,+)$ is a group and for some $\delta \geqslant 0$ a mapping $\|\cdot\|^{*}: G \rightarrow \mathbb{R}$ satisfies

$$
\left|\max \left\{\|2 x\|^{*},\|0\|^{*}\right\}-2\|x\|^{*}\right| \leqslant \delta, \quad x \in G,
$$

then, we can obtain a group-norm $\|\cdot\|: G \rightarrow \mathbb{R}$ of

$$
\max \{\|2 x\|,\|0\|\}=2\|x\|, \quad x \in G,
$$

such that

$$
-3 \delta \leqslant\|x\|-\|x\|^{*} \leqslant \delta .
$$

Also, the group-norm $\|\cdot\|$ can be written as

$$
\|x\|=\lim _{n \rightarrow \infty} \frac{1}{2^{n}}\left\|2^{n} x\right\|^{*}, \quad x \in G .
$$

By (11), $\|\cdot\|$ is uniquely determined, and by (12), $\|\cdot\|-\|\cdot\|^{*}$ is also bounded.

Proof: First, setting $x=0$ in (10) implies that $\left|\max \left\{\|0\|^{*},\|0\|^{*}\right\}-2\|0\|^{*}\right| \leqslant \delta$, therefore $\|0\|^{*} \mid \leqslant \delta$. Additionally, (10) also gives that

$$
\begin{gathered}
-\delta+2\|x\|^{*} \leqslant \max \left\{\|2 x\|^{*},\|0\|^{*}\right\} \leqslant \delta+2\|x\|^{*} \\
-\delta \leqslant\|0\|^{*} \leqslant \delta+2\|x\|^{*} \\
-\delta \leqslant\|x\|^{*}, \quad \text { for all } x \in G .
\end{gathered}
$$


Replacing $x$ with $2 x$ gives that $-\delta \leqslant\|2 x\|^{*}$, so we can obtain

$$
\begin{aligned}
& \|0\|^{*} \leqslant \delta=2 \delta-\delta \leqslant 2 \delta+\|2 x\|^{*} \\
& \|0\|^{*} \leqslant 2 \delta+\|2 x\|^{*} \quad \text { for all } x \in G .
\end{aligned}
$$

By (10) and (15), we can compute

$$
\begin{array}{ll} 
& 2\|x\|^{*} \leqslant \delta+\max \left\{\|2 x\|^{*},\|0\|^{*}\right\} \\
& 2\|x\|^{*} \leqslant \delta+\|0\|^{*} \leqslant 3 \delta+\|2 x\|^{*} \\
\text { or } & 2\|x\|^{*} \leqslant \delta+\|2 x\|^{*} \leqslant 3 \delta+\|2 x\|^{*} .
\end{array}
$$

Joining both cases, we determine that

$$
-3 \delta \leqslant\|2 x\|^{*}-2\|x\|^{*} .
$$

By (10), we can see that $\max \left\{\|2 x\|^{*},\|0\|^{*}\right\} \leqslant \delta+$ $2\|x\|^{*}$, which is possible whenever

$$
\|2 x\|^{*}-2\|x\|^{*} \leqslant \delta .
$$

Inequalities (16) and (17) imply that

$$
-3 \delta \leqslant\|2 x\|^{*}-2\|x\|^{*} \leqslant \delta \quad \text { for all } x \in G .
$$

By (18), it can be observed that the mapping $\|\cdot\|: G \rightarrow \mathbb{R}$ given in (13) exists and $\|\cdot\|$ fulfills

$$
\|2 x\|=2\|x\| \quad \text { for all } x \in G .
$$

Moreover, $\|\cdot\|$ satisfies (12). Also, replacing $x$ with $2^{n} x$ in (14) and dividing by $2^{n}$, then taking the limit $n \rightarrow \infty$ while utilizing (13), we can get that $\|x\| \geqslant 0$ for all $x \in G$. Therefore, we get (11) from (19). In view of (11) and using fact that $\|2 x\|=2\|x\| \geqslant 0$ for every $x \in G$, we can see the uniqueness of $\|\cdot\|$.

Stability results of (5) involving two variables can be easily shown with the help of Theorem 4 as follows.

Theorem 5 Suppose that $(G,+)$ is a group and a mapping $\|\cdot\|^{*}: G \rightarrow \mathbb{R}$ satisfies

$$
\left|\max \left\{\|x-y\|^{*},\|x+y\|^{*}\right\}-\|x\|^{*}-\|y\|^{*}\right| \leqslant \delta,
$$

for all $x, y \in G$ and for some $\delta \geqslant 0$. Then, there exists a unique group-norm $\|\cdot\|: G \rightarrow \mathbb{R}$ of

$$
\|x\|+\|y\|=\max \{\|x-y\|,\|x+y\|\}, \quad x, y \in G
$$

such that

$$
-3 \delta \leqslant\|x\|-\|x\|^{*} \leqslant \delta, \quad x \in G .
$$

Also,

$$
\|x\|=\lim _{n \rightarrow \infty} \frac{1}{2^{n}}\left\|2^{n} x\right\|^{*}, \quad x \in G .
$$

Proof: By Theorem 4, we can show the required stability results. First, replacing $y$ with $x$ in (20), and by Theorem 4 we can get a mapping $\|\cdot\|: G \rightarrow \mathbb{R}$. Moreover, we show that this mapping $\|\cdot\|$ fulfills (21). By replacing $x$ with $2^{n} x$ and $y$ with $2^{n} y$ in (20) and dividing by $2^{n}$, then applying the limit $n \rightarrow \infty$ and also utilizing (23), we get the required result in the form of (21).

Theorem 6 Let $(G,+)$ be a group. Assume that a mapping $\|\cdot\|^{*}: G \rightarrow \mathbb{R}$ satisfies (20), then it fulfills the proposed condition

$$
\begin{aligned}
& \lim _{n \rightarrow \infty} \frac{1}{2^{n}}\left[\max \left\{\left\|2^{n} x-2^{n} y\right\|^{*},\left\|2^{n} x+2^{n} y\right\|^{*}\right\}\right. \\
&\left.-\max \left\{\left\|2^{n}[x-y]\right\|^{*},\left\|2^{n}[x+y]\right\|^{*}\right\}\right]=0
\end{aligned}
$$

if and only if $(G,\|\cdot\|,+)$ is a normed group.

Proof: Suppose that $(G,\|\cdot\|,+)$ is a normed group, then (21) holds. Taking any elements $x, y \in G$, we have

$$
\begin{aligned}
& \mid \max \left\{\left\|2^{n} x-2^{n} y\right\|^{*},\left\|2^{n} x+2^{n} y\right\|^{*}\right\} \\
& \quad-\max \left\{\left\|2^{n}[x-y]\right\|^{*},\left\|2^{n}[x+y]\right\|^{*}\right\} \mid \\
& \leqslant\left|\max \left\{\left\|2^{n} x-2^{n} y\right\|^{*},\left\|2^{n} x+2^{n} y\right\|^{*}\right\}-\left\|2^{n} x\right\|^{*}-\left\|2^{n} y\right\|^{*}\right| \\
& \quad+\left|\max \left\{\left\|2^{n}[x-y]\right\|^{*},\left\|2^{n}[x+y]\right\|^{*}\right\}-\left\|2^{n} x\right\|^{*}-\left\|2^{n} y\right\|^{*}\right| \\
& \leqslant \delta+\left|\max \left\{\left\|2^{n}[x-y]\right\|^{*},\left\|2^{n}[x+y]\right\|^{*}\right\}-\left\|2^{n} x\right\|^{*}-\left\|2^{n} y\right\|^{*}\right| .
\end{aligned}
$$

To obtain the required statement, first dividing both sides by $2^{n}$, taking the limit $n \rightarrow \infty$, and using the proposed condition (21), we can see that

$$
\begin{aligned}
\lim _{n \rightarrow \infty} \frac{1}{2^{n}}[ & \max \left\{\left\|2^{n} x-2^{n} y\right\|^{*},\left\|2^{n} x+2^{n} y\right\|^{*}\right\} \\
& \left.\quad \max \left\{\left\|2^{n}[x-y]\right\|^{*},\left\|2^{n}[x+y]\right\|^{*}\right\}\right]=0 .
\end{aligned}
$$

Conversely, suppose that condition (24) holds. Replacing $x$ with $2^{n} x$ and $y$ with $2^{n} y$ in (20), then taking the limit $n \rightarrow \infty$ after dividing by $2^{n}$, we have

$$
\begin{aligned}
\lim _{n \rightarrow \infty} \frac{1}{2^{n}} \max \left\{\left\|2^{n} x-2^{n} y\right\|^{*},\left\|2^{n} x+2^{n} y\right\|^{*}\right\} & \\
= & \|x\|+\|y\|, \\
\lim _{n \rightarrow \infty} \frac{1}{2^{n}} \max \left\{\left\|2^{n}[x-y]\right\|^{*},\right. & \left.\left\|2^{n}[x+y]\right\|^{*}\right\} \\
& =\max \{\|x-y\|,\|x+y\|\} .
\end{aligned}
$$

By condition (24), we can compute $\|x\|+\|y\|=$ $\max \{\|x-y\|,\|x+y\|\}$.

Also, the proposed condition (24) associated with function $\|\cdot\|^{*}$ is not directly related to $(G,+)$, 
but some valuable properties about $G$ can be observed. We have given below a modified condition that is equivalent to the proposed condition (24). Consider a subsequence $m(n)$ of $\mathbb{N}$ such that

$$
\begin{aligned}
\lim _{n \rightarrow \infty} \frac{1}{2^{m(n)}}\left[\max \left\{\left\|2^{m(n)} x-2^{m(n)} y\right\|^{*},\left\|2^{m(n)} x+2^{m(n)} y\right\|^{*}\right\}\right. \\
\left.-\max \left\{\left\|2^{m(n)}[x-y]\right\|^{*},\left\|2^{m(n)}[x+y]\right\|^{*}\right\}\right]=0,
\end{aligned}
$$

which implies the mapping $\|\cdot\|^{*}$. Moreover, it holds because both of the limits

$$
\lim _{n \rightarrow \infty} \frac{1}{2^{m(n)}} \max \left\{\left\|2^{m(n)} x-2^{m(n)} y\right\|^{*},\left\|2^{m(n)} x+2^{m(n)} y\right\|^{*}\right\}
$$

and

$$
\lim _{n \rightarrow \infty} \frac{1}{2^{m(n)}} \max \left\{\left\|2^{m(n)}[x-y]\right\|^{*},\left\|2^{m(n)}[x+y]\right\|^{*}\right\}
$$

exist and are finite.

Corollary 3 Assume that a mapping $\|\cdot\|^{*}: G \rightarrow \mathbb{R}$ satisfies (11). Then it fulfills the proposed condition

$$
\begin{aligned}
& \lim _{n \rightarrow \infty} \frac{1}{2^{n}}\left[\max \left\{\left\|2^{n} x+2^{n} y\right\|^{*},\left\|2^{n} x-2^{n} y\right\|\right\}\right. \\
&\left.-\left\|2^{n}[x+y]\right\|^{*}\right]=0 \quad \text { for all } x, y \in G,
\end{aligned}
$$

if and only if $\|x\|+\|y\|=\|x+y\|$ holds for every $x, y \in G$.

Remark 1 The proposed condition (24) satisfies when $G$ is an $n$-abelian group (a group $G$ is known as an $n$-abelian group if condition $n(u+v)=n u+n v$ holds for every integer $n$ and for every $u, v \in G$, for instance, see $[1,3])$.

Remark 2 Proposed condition (24) also holds when $G$ is related to the class of groups $C_{n}$ for every natural number $n$ belongs to $\mathbb{N}\left(C_{n}\right.$ is a notation for the class of groups, which fulfills the condition $n v+n u=n u+n v$ for every $n \in \mathbb{N}$ and $u, v \in G$ ).

Remark 3 If a group-norm $\|\cdot\|$ is abelian, then the proposed condition (24) is also true.

Theorem 7 Assume that (5) is stable on a normed group $(G,\|\cdot\|,+)$. Then a free abelian group $H$ can be embedded into $G$.

Proof: Let $\|\cdot\|: G \rightarrow \mathbb{R}$ and for some $\delta \geqslant 0$, we have

$$
|\max \{\|x-y\|,\|x+y\|\}-\|x\|-\|y\|| \leqslant \delta, \quad x, y \in G .
$$

$H$ is a torsion-free group because $H$ is a free abelian group. By using the concept of HNN-extensions, for instance, see $[6,8]$. Any torsion-free group $H$ can be embedded into $G$, if for every $h \in H$, there exists an element $g \in G$ such that $g+h-g=2 h$. If $H$ is embedding into $G$, then (26) implies that

$$
|\max \{\|h+g\|,\|h-g\|\}-\|h\|-\|g\|| \leqslant \delta, \quad h, g \in G .
$$

It will be shown that $\|\cdot\|$ is bounded. The proof is obvious when $\delta=0$, so assume that $\delta>0$. More explicitly, we show that $\|h\|<2 \delta$ for every $h \in G$. By (27), we have two possible values that either $\mid \| h-$ $g\|-\| h\|-\| g \| \mid \leqslant \delta$ or $|\|h+g\|-\|h\|-\|g\|| \leqslant \delta$. Considering the first possibility and setting $h=g=$ 0 , we can see that $\|0\| \leqslant \delta$. Setting $g=h$, we can conclude that

$$
\begin{aligned}
|\|0\|-2\|h\|| & \leqslant \delta \\
|2\|h\||-\||\|0\|| & \leqslant \delta \\
2\|h\| & \leqslant \delta+\|0\| \\
2\|h\| & \leqslant \delta+\delta \\
\|h\| & \leqslant \delta .
\end{aligned}
$$

For the second possibility, we can obtain

$$
|\|h+g\|-\|h\|-\|g\|| \leqslant \delta .
$$

On the contrary, assume that $\|h\| \geqslant 2 \delta$ for some $h \in G$. Setting $g=h$ in (28), we have

$$
\begin{aligned}
|\|2 h\|-2\|h\|| & \leqslant \delta \\
|2\|h\|-\|2 h\|| & \leqslant \delta \\
2\|h\|-\delta & \leqslant\|2 h\| \\
3 \delta & \leqslant\|2 h\| .
\end{aligned}
$$

Again, setting $g=2 h$ in (28) we have

$$
\begin{aligned}
|\|3 h\|-\|h\|-\|2 h\|| & \leqslant \delta \\
|\|h\|+\|2 h\|-\|3 h\|| & \leqslant \delta \\
|\|h\||+|\|2 h\|| & \leqslant \delta+\|3 h\| \\
5 \delta-\delta & \leqslant\|3 h\| \\
4 \delta & \leqslant\|3 h\| .
\end{aligned}
$$

Repeating this process for $g=3 h$, we can conclude $\|4 h\| \geqslant 4 \delta$. Continuing the process, we can determine

$$
(m+1) \delta \leqslant\|m h\|
$$

where $m=1,2, \ldots$, so we can see that $\|m h\|$ is unbounded when the value of $m$ varies.

Also, let $g \in G$ such that $2 h=g+h-g$. Then $2 m h=g+m h-g$ for any integer $m>0$. Moreover, for any $m$, setting $g=m h$ and $h=m h$ in (28), we have

$$
\begin{array}{r}
|\|2 m h\|-2\|m h\|| \leqslant \delta \\
|\|g+m h-g\|-2\|m h\|| \leqslant \delta .
\end{array}
$$


Moreover, (28) follows that

$$
\begin{gathered}
|\|g+m h-g\|-\|g\|-\|m h-g\|| \leqslant \delta \quad \text { and } \\
|\|m h-g\|-\|m h\|-\|-g\|| \leqslant \delta ;
\end{gathered}
$$

thus, we can get

$$
\begin{aligned}
\mid\|g+m h-g\| & -\|g\|-\|m h\|-\|-g\| \mid \\
\leqslant & |\|g+m h-g\|-\|g\|-\|m h-g\|| \\
& +|\|m h-g\|-\|m h\|-\|-g\|| \\
\leqslant & 2 \delta
\end{aligned}
$$

From (29) and (30) we have

$$
\begin{aligned}
& |\|g+m h-g\|-2\|m h\|+\|m h\|| \leqslant 2 \delta+\|g\|+\|-g\| \\
& \quad\|m h\| \leqslant 2 \delta+\|g\|+\|-g\|+|\|g+m h-g\|-2\|m h\|| \\
& \|m h\| \leqslant 5 \delta,
\end{aligned}
$$

for $m=1,2, \ldots$, which is a contradiction. This completes the proof because the group-norm $\|\cdot\|$ is bounded.

Corollary 4 Assume that (5) is stable on a normed $\operatorname{group}(G,\|\cdot\|,+)$, and $H$ is a discretely normed abelian group. Then $H$ is embedding into $G$.

Proof: Since $H$ is a discretely normed abelian group, then $H$ is a free group, for instance, see [11]; consequently $H$ is embedding into $G$.

When we analyzed condition (24), it is noticed that for the stability of (5), the given condition (24) is necessary and sufficient. This condition leads to the following definition.

Definition 3 ([16]) A group $(G,+)$ is called weakly commutative if for any $a, b \in G$, there exists $n=$ $n(a, b) \geqslant 2$ such that $2^{n}(a+b)=2^{n} a+2^{n} b$.

When we consider Theorem 6 and Definition 3 about Tabor weakly commutativity, then it gives the following theorem.

Theorem 8 Let $\left(G,\|\cdot\|^{*},+\right)$ be a Tabor weakly commutative, then the group-norm $\|\cdot\|$ satisfies (5).

Proof: From Theorem 6, it can be seen that condition (24) satisfies when $G$ is weakly commutative. To prove the second condition presented in Theorem 6 , we need to construct a sequence $\{m(n)\}$, which holds the second condition. For this purpose, assume that $m_{1}=n(x, y)$ for fixed $x, y \in G$. Considering the pair $\left(2^{m_{1}} x, 2^{m_{1}} y\right)$, by our assumption, there exists $n\left(2^{m_{1}} x, 2^{m_{1}} y\right)$ such that

$$
\begin{aligned}
2^{n\left(2^{m_{1}} x, 2^{m_{1}} y\right)}\left(2^{m_{1}} x+2^{m_{1}} y\right) \\
=2^{n\left(2^{m_{1}} x, 2^{m_{1}} y\right)}\left(2^{m_{1}} x\right)+2^{n\left(2^{m_{1}} x, 2^{m_{1}} y\right)}\left(2^{m_{1}} y\right) .
\end{aligned}
$$

Since $2^{m_{1}}(x+y)=2^{m_{1}} x+2^{m_{1}} y$, so we get that

$$
\begin{aligned}
2^{m_{1}+n\left(2^{m_{1}} x, 2^{m_{1}} y\right)}(x+y) & \\
= & 2^{m_{1}+n\left(2^{m_{1}} x, 2^{m_{1}} y\right)} x+2^{m_{1}+n\left(2^{m_{1}} x, 2^{m_{1}} y\right)} y .
\end{aligned}
$$

Again, assume that $m_{1}+n\left(2^{m_{1}} x, 2^{m_{1}} y\right)=m_{2}$; therefore, we have $2^{m_{2}}(x+y)=2^{m_{2}} x+2^{m_{2}} y$. By mathematical induction, it leads to the required sequence $\left\{m_{n}\right\}$.

Acknowledgements: This work was supported by the National Natural Science Foundation of China [Grant number 11971493 and 12071491].

\section{REFERENCES}

1. Alperin JL (1969) A classification of $n$-abelian groups. Can J Math 21, 1238-1244.

2. Bingham N, Ostaszewski A (2010) Normed versus topological groups: Dichotomy and duality. Diss Math 472, 1-138.

3. Baer R (1953) Factorization of $n$-soluble and $n$ nilpotent groups. Proc Am Math Soc 4, 15-26.

4. Chaljub-Simon A, Volkmann P (1994) Caractérisation du module d'une fonction additive à l'aide d'une équation fonctionnelle. Aequ Math 47, 60-68.

5. Gilanyi A, Nagatou K, Volkmann P (2010) Stability of a functional equation coming from the characterization of the absolute value of additive functions. Ann Funct Anal 1, 1-6.

6. Higman G, Neumann BH, Neuman H (1949) Embedding theorems for groups. $J$ London Math Soc 1, 247-254.

7. Kannappan Pl (1968) The functional equation $f(x y)+f\left(x y^{-1}\right)=2 f(x) f(y)$ for groups. Proc Amer Math Soc 19, 69-74.

8. Magnus W, Karrass A, Solitar D (2004) Combinatorial Group Theory: Presentations of Groups in Terms of Generators and Relations, 2nd Edn, Dover Publications, USA.

9. Przebieracz B (2011) The stability of functional equation $\min \{f(x+y), f(x-y)\}=|f(x)-f(y)|$. J Inequal Appl 2011, ID 22.

10. Redheffer RM, Volkmann P (1997) Die Funktionalgleichung $f(x)+\max \{f(y), f(-y)\}=$ $\max \{f(x+y), f(x-y)\}$, International Series of Numerical Mathematics 123, Birkhauser: Basel, Switzerland.

11. Steprāns J (1985) A characterization of free abelian groups. Proc Am Math Soc 93, 347-349.

12. Sarfraz M, Liu Q, Li Y (2020) Stability of maximum functional equation and some properties of groups. Symmetry 12, ID 1949. 
13. Sarfraz M, Ali F, Li Y (2020) Lipschitz isomorphism and fixed point theorem for normed groups. Cogent Math Stat 7, ID 1859673.

14. Toborg I (2017) On the functional equation $f(x)+$ $f(y)=\max \left\{f(x y), f\left(x y^{-1}\right)\right\}$ on groups. Arch Math 109, 215-221.
15. Volkmann P (2017) Charakterisierung des Betrages Reellwertiger Additiver Funktionen auf Gruppen, KITopen.

16. Tabor J (1985) Remark 18, 22nd international symposium on functional equations, Oberwolfach 1984. Aequ Math 29, ID 96. 